\title{
Análisis de las variables sociales en los actos de habla de petición en chino y en español
}

\section{Analysis of Social Variables in the Speech Acts of Petition in Chinese and Spanish}

DOI: 10.32870/mycp.v10i28.699

Xinghua Li ${ }^{1}$

\begin{abstract}
Resumen
Basándose en un corpus literario, este estudio tiene como objetivo analizar los aspectos contrastivos de la cortesía verbal en chino y en español y comprobar si las variables sociales influyen o no sobre la realización de la petición en las dos lenguas. Según los análisis, hemos conseguido los siguientes resultados: 1) desde el punto de vista de las estrategias de actos principales, casi independientemente de las variables sociales, en ambas lenguas se presenta la directividad y la preferencia de uso de las estrategias directas en la realización de la petición; 2) en chino, a pesar de la variación de la combinación de las variables sociales, el uso de las modificaciones internas y externas es habitual; sin embargo, en español se muestra igualmente un uso reducido; 3) en comparación con el español, en chino se utilizan con más frecuencia los marcadores de apertura.
\end{abstract}

Palabras clave: pragmática contrastiva, comunicación intercultural, actos de habla de petición, variables sociales, cortesía verbal.

\begin{abstract}
Based on the literary corpus, this study aims to analyze the contrastive aspects of verbal politeness both in Chinese and Spanish and determine whether social variables have influence on the realization of the request in both languages. According to the analysis, we have achieved the following results: 1) Given the strategies of the head act, no matter the social variables or the situations, in both languages present directness and the preference of using direct strategies in carrying out the request 2) In Chinese, despite the variation of the combination of social variables, the use of internal and external modifications is habitual; however, a reduced use has presented in English 3) Compared with Spanish, openers are more frequently used in Chinese.
\end{abstract}

Keywords: contrastive pragmatics, intercultural communication, speech acts of request, social variables, verbal courtesy.

Sabemos que en los años sesenta comenzó el verdadero estudio de la pragmática, ámbito que fue definido por Leech (1983: 6) como "the study of meaning in relation to speech situations". En los años ochenta varios estudios realizados demostraron la existencia de características específicas del discurso en lenguas de culturas determinadas, lo cual dio comienzo al desarrollo del ámbito de estudio conocido como pragmática intercultural. Los primeros estudios que

Artículo recibido el 05 de mayo de 2020 y dictaminado el 13 de junio de 2020.

1. Universidad de Nankai, 9Weijin Road, Tianjin 300071, P. R. China. ORCID: https://orcid.org/00000002-0194-3508 Correo electrónico: 516965366@qq.com 
se centran en este tema son el de Kitagawa (1980), que compara asentimientos en inglés y japonés; el de Coulmas (1981), que contrasta expresiones de agradecimiento y peticiones de disculpas en japonés y una serie de lenguas europeas; el de Amzilotti (1983), que analiza la producción de preguntas retóricas en inglés e italiano, etc. Estos estudios y los posteriores como los de Bintini (2013) y Tziyu (2015) confirman que las reglas lingüísticas según una determinada sociedad no coinciden con las según otra; en consecuencia puede dar lugar a diferencias socioculturales en el uso de la lengua.

De este modo, esta disciplina llama cada día más atención de los investigadores, ya que la escasez o ausencia de competencia pragmática por parte de los aprendices de lenguas extranjeras puede conducir a interacciones verbales erróneas e inapropiadas, e incluso provocar choques comunicativos.

En el presente estudio hemos decidido analizar la influencia de las variables sociales en la realización de los actos de habla de petición en chino y en español, debido a que éstos se producen con mayor frecuencia en la comunicación interpersonal, y que en gran medida las investigaciones se centran principalmente en comparar el chino y el inglés, motivo por el que este trabajo puede considerarse pionero.

\section{Diseño situacional}

Hemos escogido cuatro obras españolas y siete obras chinas ${ }^{2}$ para la elaboración del corpus que será instrumento metodológico de investigación para la recopilación de datos. La elección de las novelas tiene en consideración factores como: traducción — sirve para la posible mal interpretación de los actos de habla por parte de la propia autora, debido a la falta de la competencia en dicha lengua - , estilo narrativo, frecuencia de producción de los actos de habla, verosimilitud y naturalidad de actos de habla, etcétera.

Para el análisis situacional emplearemos las siguientes variables: distancia social, poder relativo y grado de imposición, diferenciándolas entre distintos niveles, según el contexto y la relación interpersonal de las novelas. De este modo se puede ofrecer un marco para comparar diferencias transculturales en la realización de la petición.

2. Las informaciones bibliográficas de las obras están situadas en el apartado de referencias. 
1. Distancia social: hace referencia a la familiaridad que atañe al grado de conocimiento previo y al grado de empatía. En este sentido, cuanto mayor es la familiaridad, menor será la distancia social.

a. Distancia social (+): entre personajes desconocidos que tienen estatus igual/desigual y que han tenido ninguna o poca relación social, existe la mayor distancia social, como en el caso de autoridades y subordinados, dependientes de establecimientos y clientes, etcétera.

b. Distancia social (=): entre personajes conocidos que tienen estatus igual/desigual, existe una distancia social neutra, por ejemplo: amigos, colegas, compañeros, etcétera.

c. Distancia social (-): entre personajes muy conocidos que tienen estatus igual/desigual, existe poca o ninguna distancia social, como en el caso de familiares, novios, matrimonios, etcétera.

2. Grado de imposición: está relacionado con el del esfuerzo que se requiere, es decir, el del coste que supone realizar el acto pedido.

a. Grado de imposición normal (G+): al oyente le supone un bajo coste mental, económico, físico, etc., llevar a cabo el acto pedido, como es el caso del siguiente ejemplo, en el que el hablante pide al oyente que no se mueva para no ahuyentar al conejo:

Ya lo veo. No os mováis, por favor, no os mováis, que ya es mio (Sánchez, 2006, p. 296).

b. Grado de imposición alto (G++): al oyente le supone un alto coste mental, económico, físico, etc., llevar a cabo el acto pedido. En el ejemplo que aportamos el hablante pide al receptor que libere a su hijo, que ha sido detenido por las autoridades:

把 我-的孩-子放下来, 一-切由我承-担。

Bă wǒ-de hái-zi fàng xiàlái, yí-qiè yóu wǒ chéng-dān.

Suelta a mi niño, voy a ser responsable de todo (Yan, 2012b, p. 356).

3. Poder relativo: esta variable social nunca es estable, ya que tiene que ver con el control material o no material del oyente sobre el hablante (Brown \& Levinson, 1994, p. 77). En consecuencia, clasificaremos dos tipos de poder relativo, en consideración de la relación interpersonal, de las situaciones contextuales en que se lleva a cabo el acto pedido y de los tipos de petición. 
a. A. El poder relativo que existe entre los interlocutores es simétrico $(H$ $=0$ ). En tales situaciones, debido a que el efecto para llevar a cabo el acto pedido afecta menos de manera negativa al hablante, el oyente muestra un grado de control bajo sobre el acto pedido por el hablante.

\section{Tabla 1}

Poder relativo simétrico $(\mathrm{H}=\mathrm{O})$

\begin{tabular}{l|l}
\hline Tipos de actos pedidos & Ejemplos \\
\hline $\begin{array}{l}\text { Peticiones que no interrumpen en exceso la actividad } \\
\text { habitual del oyente, como esperar a alguien, solicitar su } \\
\text { compañía cuando está libre el hablante, darse prisa, etc. }\end{array}$ & $\begin{array}{l}\text { Espera un momento, déjame } \\
\text { decirte una palabra más (Yu, } \\
\text { 2013, p.18). }\end{array}$ \\
\hline $\begin{array}{l}\text { Peticiones para animar el ambiente, pedir información (no } \\
\text { privada ni importante): preguntar la hora, una dirección, } \\
\text { etc. }\end{array}$ & $\begin{array}{l}\text { Hermana mayor, cuéntame } \\
\text { chistes (Yu, 2013, p. 8). }\end{array}$ \\
\hline $\begin{array}{l}\text { Peticiones para transmitir mensajes, recados, avisos, etc., } \\
\text { o para poner al alcance algo material como un libro, vaso, } \\
\text { etc. }\end{array}$ & $\begin{array}{l}\text { Avísale de mi parte que tengo } \\
\text { algo que hablar con él (Yu, } \\
\text { 2013, p. 22). }\end{array}$ \\
\hline $\begin{array}{l}\text { Peticiones que resultan indiferentes para el oyente o son } \\
\text { meramente corteses, como pedir permiso, conversación, } \\
\text { estar en paz, etc. }\end{array}$ & $\begin{array}{l}\text { Déjeme subir (Yu, 2013, p. } \\
\text { 26). }\end{array}$ \\
\hline $\begin{array}{l}\text { Peticiones para guardar información cuyo contenido no } \\
\text { está relacionado con el oyente, o no le importa o afecta } \\
\text { demasiado: guardar información de los demás. }\end{array}$ & $\begin{array}{l}\text { Papá, de todas maneras, que no } \\
\text { se entere de que trabajo aquí } \\
\text { (Yu, 2013, p. 31). }\end{array}$ \\
\hline
\end{tabular}

Fuente: elaboración propia.

b. B. El oyente tiene el poder relativo sobre el hablante $(\mathrm{H}<\mathrm{O})$. El oyente puede mostrar un alto grado de control sobre el acto pedido por el hablante, pues, al llevar a cabo el acto pedido, su efecto le puede afectar más de manera negativa. 
Análisis de las variables sociales en los actos de habla de petición en chino y en español

\section{Tabla 2}

Poder relativo del oyente sobre el hablante $(\mathrm{H}<\mathrm{O})$

\begin{tabular}{l|l}
\hline Tipos de actos pedidos & Ejemplos \\
\hline $\begin{array}{l}\text { Peticiones que pueden crear problemas al oyente: } \\
\text { pedirle que libere a presos, que haga trampas en una } \\
\text { selección política, etc. }\end{array}$ & $\begin{array}{l}\text { Dile a tus superiores que le dejen en } \\
\text { libertad (Yan, 2012a, p. 245). }\end{array}$ \\
\hline $\begin{array}{l}\text { Peticiones para modificar lo creído y pensado: pedir } \\
\text { a alguien que no hable de cierta manera, que cambie } \\
\text { de creencias, de opiniones, etc. }\end{array}$ & $\begin{array}{l}\text { Papá, estás loco por la pobreza, no } \\
\text { digas disparates (Yu, 2013, p. 16). }\end{array}$ \\
\hline $\begin{array}{l}\text { Peticiones que conllevan la intención de meterse en } \\
\text { asuntos o trabajos ajenos. }\end{array}$ & $\begin{array}{l}\text { Lustre mejor esos zapatos del señor } \\
\text { (Yu, 2013, p. 16). }\end{array}$ \\
\hline $\begin{array}{l}\text { Peticiones para manipular algo que está en posesión } \\
\text { del oyente: dejar a alguien mirar, observar, tocar, } \\
\text { utilizar, etc., cosas que pertenecen al interlocutor. }\end{array}$ & $\begin{array}{l}\text { Déjame utilizar tu mochila (ejemplo } \\
\text { propio). }\end{array}$ \\
\hline $\begin{array}{l}\text { Peticiones para controlar una emoción, un estado } \\
\text { psicológico o mental: pedir al oyente que ría o llore } \\
\text { por algo. }\end{array}$ & $\begin{array}{l}\text { Hija, no me reproches que tenga un } \\
\text { corazón duro, tengo mis razones que } \\
\text { me atormentan sin poder hablar de } \\
\text { eso (Yu, 2013, p. 161). }\end{array}$ \\
\hline $\begin{array}{l}\text { Peticiones que están en contra de la voluntad del } \\
\text { oyente. }\end{array}$ & $\begin{array}{l}\text { Vuelve, quiero que te sientes un } \\
\text { momento (Yu, 2013, p. 63). }\end{array}$ \\
\hline
\end{tabular}

Fuente: elaboración propia.

\section{Codificación de la petición}

Como señala en la tabla 3, para el análisis codificamos los ejemplos de peticiones, 744 ejemplos de peticiones en chino y 673 en español, en 12 situaciones, en función de la diversidad de la combinación de las variables. 
Tabla 3

Diseño situacional

\begin{tabular}{|c|c|c|c|}
\hline \multirow[t]{2}{*}{ Situaciones } & \multirow{2}{*}{$\begin{array}{c}\text { Combinación de las } \\
\text { variables sociales }\end{array}$} & \multicolumn{2}{|c|}{ Número de actos de habla } \\
\hline & & Chino & Español \\
\hline S.1 & $\mathrm{D}+, \mathrm{H}=\mathrm{O}, \mathrm{G}+$ & 29 & 63 \\
\hline S.2 & $\mathrm{D}+, \mathrm{H}=\mathrm{O}, \mathrm{G}++$ & 10 & 10 \\
\hline S.3 & $\mathrm{D}+, \mathrm{H}<\mathrm{O}, \mathrm{G}+$ & 62 & 41 \\
\hline S.4 & $\mathrm{D}+, \mathrm{H}<\mathrm{O}, \mathrm{G}++$ & 99 & 41 \\
\hline S.5 & $\mathrm{D}=, \mathrm{H}=\mathrm{O}, \mathrm{G}+$ & 32 & 107 \\
\hline S.6 & $\mathrm{D}=, \mathrm{H}=\mathrm{O}, \mathrm{G}++$ & 13 & 24 \\
\hline S.7 & $\mathrm{D}=, \mathrm{H}<\mathrm{O}, \mathrm{G}+$ & 71 & 110 \\
\hline S.8 & $\mathrm{D}=, \mathrm{H}<\mathrm{O}, \mathrm{G}++$ & 60 & 67 \\
\hline S.9 & $\mathrm{D}-, \mathrm{H}=\mathrm{O}, \mathrm{G}+$ & 64 & 65 \\
\hline S.10 & $\mathrm{D}-, \mathrm{H}=\mathrm{O}, \mathrm{G}++$ & 13 & 10 \\
\hline S.11 & $\mathrm{D}-, \mathrm{H}<\mathrm{O}, \mathrm{G}+$ & 103 & 86 \\
\hline S.12 & $\mathrm{D}-, \mathrm{H}<\mathrm{O}, \mathrm{G}++$ & 188 & 49 \\
\hline Total de los actos de habla & & 744 & 673 \\
\hline
\end{tabular}

Fuente: elaboración propia.

Debido a que nuestro corpus se basa en obras literarias, la cifra de ejemplos de petición que aparecen en cada situación no es controlable. Efectivamente, como se ve en la tabla anterior, en algunos casos se han encontrado más de cien actos de petición, y sin embargo en otros sólo 10. Para solucionar este problema realizaremos tanto el análisis cuantitativo como el estadístico, es decir, el análisis de la frecuencia de uso y la prueba estadística, y de este modo los resultados serán más precisos.

Para el análisis estadístico aplicaremos el programa estadístico “(Statistical Package for the Social Sciences (SPSS)). Mediante la prueba Mann-Whitney U, que es una prueba no paramétrica aplicada a dos muestras independientes (chino y español), podemos comprobar si existen diferencias significativas entre el chino y el español, comparando los promedios de las estrategias aplicadas en los actos de habla de petición. Los niveles significativos se determinan entre ${ }^{*} \mathrm{p}<0.05,{ }^{* *} \mathrm{p}<0.01 \mathrm{y}{ }^{* * *} \mathrm{p}<0.001$. Cuanto menor sea el valor probabilístico, más diferencias estadísticas se mostrarán en el análisis. 


\section{Resultados del análisis}

El estudio de Rue y Zhang (2008) realiza una codificación de las peticiones muy detallada, las analiza desde el punto de vista de sus tres constituyentes: 1) actos principales; 2) movimientos de apoyo, y 3) marcadores de apertura, lo cual nos servirá de modelo para nuestro análisis.

\subsection{Actos principales}

Dependiendo del nivel de directividad, las estrategias utilizables en los actos principales se pueden clasificar en tres tipos.

\section{Tabla 4}

Clasificación de las estrategias según la directividad

\begin{tabular}{l|l|l}
\hline Estrategias & Características & Subestrategias representativas \\
\hline Estrategias directas & $\begin{array}{l}\text { El acto de habla se realiza por } \\
\text { medio de la modalidad oracional } \\
\text { convencionalmente asociada con } \\
\text { su fuerza ilocutiva. }\end{array}$ & $\begin{array}{l}\text { 1. Modo imperativo: Cierra la } \\
\text { puerta. } \\
\text { 2. Declaraciones de obligación: } \\
\text { Tienes que cerrar la puerta. } \\
\text { 3. Declaraciones de deseo: Quiero } \\
\text { que cierres la puerta. } \\
\text { 4. Performativos: Te ruego que } \\
\text { cierres la puerta. }\end{array}$ \\
\hline $\begin{array}{l}\text { Estrategias } \\
\text { convencionalmente } \\
\text { indirectas }\end{array}$ & $\begin{array}{l}\text { La convención del contenido } \\
\text { proposicional y las formas } \\
\text { lingǘsticas se combinan para } \\
\text { indicar su fuerza ilocutiva. }\end{array}$ & $\begin{array}{l}\text { 1. Fórmulas de sugerencia: ¿No } \\
\text { sería bueno cerrar la puerta? } \\
\text { 2. Preparadores de interrogación: } \\
\text { ¿Puedes cerrar la puerta? }\end{array}$ \\
\hline $\begin{array}{l}\text { Estrategias no } \\
\text { convencionalmente } \\
\text { indirectas }\end{array}$ & $\begin{array}{l}\text { El conocimiento compartido de } \\
\text { las personas que interactúan } \\
\text { determina la fuerza ilocutiva del }\end{array}$ & $\begin{array}{l}\text { 1. Indicio fuerte: Has dejado tu } \\
\text { habitación muy desordenada. } \\
\text { 2. Indicio débil: No puedo encontrar } \\
\text { mis libros. } \\
\text { (El hablante pide al oyente que } \\
\text { recoja la habitación). }\end{array}$ \\
\hline
\end{tabular}

Fuente: elaboración propia.

Como demuestra la figura 1 , el resultado indica que las estrategias directas presentan un uso mayoritario sin ningún tipo de exclusión. El porcentaje mínimo que éstas ocupan son del $81.8 \%$ en la $\mathrm{S} .4(\mathrm{D}+, \mathrm{H}<\mathrm{O}, \mathrm{G}++)$ y del $71.4 \%$ en la $S .1(D+, H=O, G+)$ en chino y en español, respectivamente, y tanto en 
chino como en español prefieren los interlocutores el uso del modo imperativo, seguido de los preparadores. Podemos ver que, a pesar de la variación situacional, en ambas lenguas se muestra un alto nivel de directividad a la hora de llevar a cabo el acto de habla de petición.

Por otro lado, podemos notar que en la S.4 (D+, H<O, G++), el uso de las estrategias directas llega al mínimo porcentaje $-81.8 \%$ - en chino y al penúltimo - 73.1\% - en español; en las $S .3(\mathrm{D}+, \mathrm{H}<\mathrm{O}, \mathrm{G}+)$ y $\mathrm{S} .8(\mathrm{D}=, \mathrm{H}<\mathrm{O}$, $\mathrm{G++}$ ), también se encuentra un menor uso de ellas en ambas lenguas. Según Brown y Levinson (1987), cada individuo tiene y reclama para sí una cierta imagen pública ${ }^{3}$ que quiere conservar, ya que es algo tan vulnerable que todos tratan de protegerla. De acuerdo con el modelo de estos autores: $W=D(S, H)$ $+P(H, S)+R x^{4}$, el riesgo potencial de (Actos de Habla Amenazantes Para la Imagen (FTA)) en tales situaciones es relativamente más alto que otras. En consecuencia, podemos llegar a la conclusión de que, cuanto más alto es el riesgo potencial del FTA, menos directividad de los actos principales se mostrará en ambas lenguas. Esto nos demuestra que los interlocutores de ambas lenguas tienen en cuenta la imagen negativa en la producción de la petición.

3. Según los autores, hay dos tipos de imágenes:

Imagen negativa (negative face): deseo de tener libertad de acción, de no sufrir imposiciones por parte de los demás y de dominar el propio territorio.

Imagen positiva (positive face): deseo de ser apreciado por los demás y de que otros compartan los mismos deseos.

4. W: la gravedad del acto contra la imagen.

$\mathrm{P}(\mathrm{H}, \mathrm{S})$ : poder relativo (power) del destinatario respecto al emisor.

D (S, H): distancia social (distance), influye sobre el grado de familiaridad y contacto entre los interlocutores.

Rx: grado de imposición (ranking of imposition) de un determinado acto respecto a la imagen pública. 


\section{Figura 1}

Frecuencia de uso de las estrategias directas

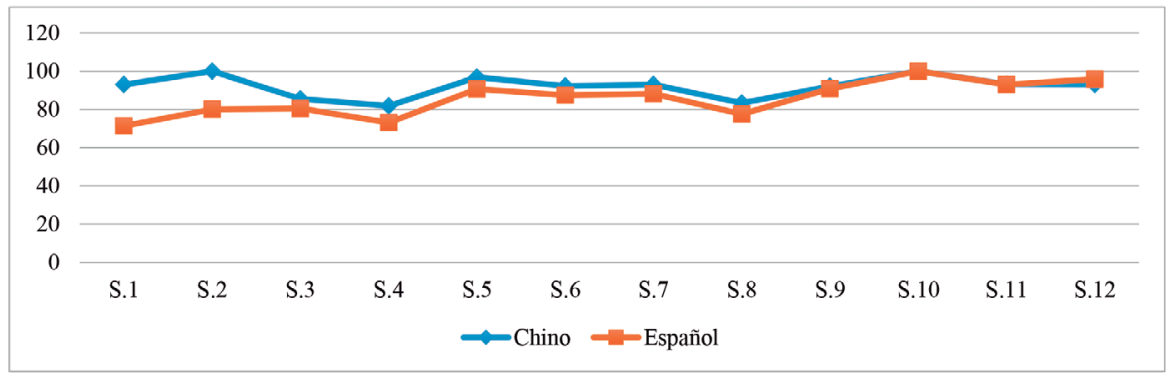

Fuente: elaboración propia.

Por otra parte, en comparación con el español, el chino presenta una tendencia de mayor uso de estrategias directas, salvo en las $\mathrm{S} .10$ (D-, $\mathrm{H}=\mathrm{O}, \mathrm{G}++$ ) y $\mathrm{S} .12$ (D-, $\mathrm{H}<\mathrm{O}, \mathrm{G}++$ ), aunque este contraste sólo resulta estadísticamente significativo en la $\mathrm{S} .1(\mathrm{D}+, \mathrm{H}=\mathrm{O}, \mathrm{G}+)(\mathrm{p}=0.020)$. En cuanto al uso de estrategias concretas, las diferencias significativas se detectan en muy pocos casos: en chino se utilizan menos formulaciones de sugerencia en la S.4 (D+, H<O, G++) $(\mathrm{p}=0.042)$, y más el modo imperativo en la $\mathrm{S} .8(\mathrm{D}=, \mathrm{H}<\mathrm{O}, \mathrm{G}++)(\mathrm{p}=0.047)$.

Para que el análisis sea más completo, también hemos examinado la sensibilidad a las variables sociales. En primer lugar, hemos clasificado los ejemplos de petición del corpus según los diferentes niveles de la distancia social, el grado de imposición y el poder relativo, respectivamente. Las cifras de actos de habla correspondientes a cada caso están expuestas en la siguiente tabla.

\section{Tabla 5}

Distribución de los actos de habla según los niveles de las variables sociales

\begin{tabular}{l|l|c|c|c|c|c|c|c}
\hline & & \multicolumn{4}{|c|}{ Distancia social } & \multicolumn{2}{c|}{$\begin{array}{c}\text { Grado de } \\
\text { imposición }\end{array}$} & \multicolumn{2}{c}{ Poder relativo } \\
\hline & & $D++$ & $D+$ & $D-$ & $G+$ & $G++$ & $H=0$ & $H<0$ \\
\hline $\begin{array}{l}\text { Número } \\
\begin{array}{l}\text { de actos de } \\
\text { habla }\end{array}\end{array}$ & Chino & 200 & 176 & 368 & 361 & 383 & 161 & 583 \\
\cline { 2 - 9 } & Español & 155 & 308 & 210 & 472 & 201 & 279 & 394 \\
\hline
\end{tabular}

Fuente: elaboración propia. 
Según la frecuencia de uso, sea como sea el nivel de las variables sociales, la utilización de las estrategias directas es mayoritaria. Cuando la distancia social es mayor, en ambas lenguas se observa un uso de estrategias directas reducido y un aumento de estrategias convencionalmente indirectas que cuando ésta es menor ( $\mathrm{p}=.009, \mathrm{p}=.000$ en chino y español, respectivamente). Por otro lado, sólo en español se nota que cuando la distancia social es mayor, los interlocutores utilizan menos estrategias directas y más estrategias convencionalmente indirectas que cuando ésta es neutra $(\mathrm{p}=.002)$. Por eso, tanto en chino como en español se considera la distancia social en la realización de la petición, pero en español se muestra mayor sensibilidad a esta variable. En cuanto al poder relativo y el grado de imposición, no hemos detectado ningún resultado de muestra de la sensibilidad.

De acuerdo con los resultados anteriores, se observa la misma preferencia de uso de estrategias directas en ambas lenguas, a pesar de las diferencias confirmadas por las pruebas estadísticas. Según Brown y Levinson (1987), para proteger la imagen del oyente en los actos de habla de petición, los interlocutores deberían recurrir más a estrategias menos directas. Sin embargo, el resultado que hemos obtenido contradice evidentemente esta teoría.

Esta paradoja se debe a la relación entre cultura y cortesía verbal. China confiere una importancia capital a los valores colectivistas, a vínculos sociales fuertes y a unas relaciones interpersonales basadas en el grupo y la cooperación. A priori, los miembros de dicha cultura tienden a establecer una relación de confianza e intimidad en la comunicación interpersonal. Frente a un interlocutor del mismo grupo, que comparte edad o estatus social similares, etc., se suelen emplear estrategias directas, mostrando de esta manera el deseo de lograr la confianza del receptor por parte del hablante. Se ha de tener en cuenta, asimismo, que debido a la influencia del antiguo sistema político, de carácter fuertemente jerárquico, las relaciones interpersonales en la cultura china son asimétricas. En consecuencia, el empleo de estrategias directas por un interlocutor de categoría alta (edad, estatus social, rango, etc.) hacia uno de categoría baja se considera normal y no un signo de descortesía.

La cultura española, si bien tiende a ser más individualista, se caracteriza por el acercamiento, es decir, las interacciones se basan en y están marcadas por la solidaridad, como afirma Briz (2010). En su cultura la gente estrecha generalmente en seguida los espacios interpersonales, tiende a construir espacios comunes con el otro y nivela las diferencias sociales y funcionales, mostrando una relación simétrica (Briz, 2010, pp.12-13). Esta característica 
favorece la menor frecuencia de atenuantes y un estilo comunicativo más coloquial, por lo que en culturas de acercamiento, un acto de habla de petición directo será más común y no se considerará una amenaza para la imagen.

Aunque las culturas china y española son distintas en sus características, lo curioso es que esas diferencias han producido cierta similitud en la preferencia del uso de estrategias directas en la realización de la petición.

En cuanto a las modificaciones internas que forman parte de los actos principales, sirven para minimizar o maximizar la fuerza ilocutiva del acto pedido. Su uso ha sobrepasado el $50.0 \%$ en todas las situaciones, salvo en la S.7 $(\mathrm{D}=, \mathrm{H}<\mathrm{O}, \mathrm{G}+)$; a diferencia del chino, en español sólo llega a esta cifra en la $S .1(D+, H=O, G+)$, como se ve en la siguiente figura. De esto podemos deducir que los interlocutores mitigan con más frecuencia la fuerza ilocutiva mediante las modificaciones en chino que en español. Además, vemos que cuando la distancia social es mayor, se utilizan más modificaciones internas que cuando ésta es neutra o menor, tanto en chino como en español. Por eso, su uso es bastante sensible a la distancia social en ambas lenguas. En cuanto a la sensibilidad a otras variables, cuando el grado de imposición es normal, los hablantes de chino aplican menos modificaciones internas a la petición que cuando éste es alto $(\mathrm{p}=.014)$, por lo que se puede afirmar que éstas son sensibles al grado de imposición sólo en chino.

\section{Figura 2}

Frecuencia de uso de las modificaciones internas

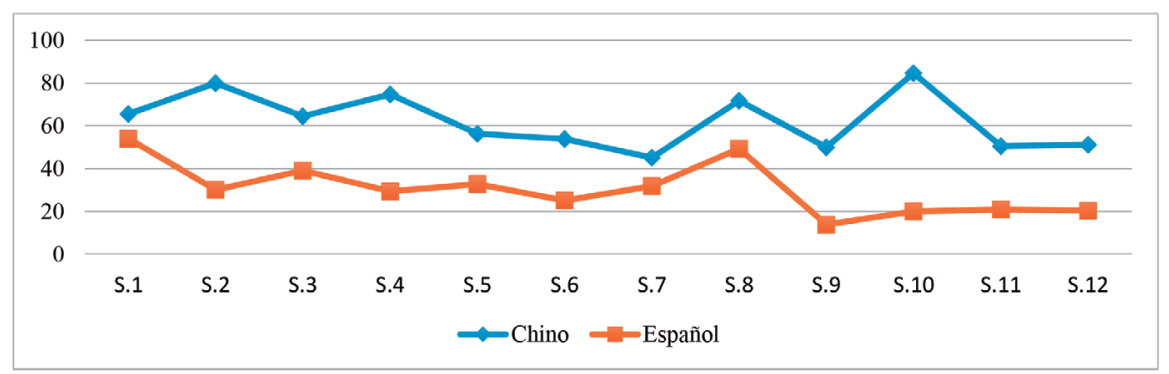

Fuente: elaboración propia.

Por otro lado, según las pruebas estadísticas, las estrategias que se emplean con más frecuencia en chino son los minimizadores y los marcadores pragmáticos en las dos situaciones señaladas. En español, por su parte, el uso de 
los marcadores de cortesía, de la reiteración de la petición y de las estructuras con condicionales es más frecuente, si lo comparamos con el chino, en la mitad de las situaciones indicadas en la tabla 3.

\section{Tabla 6}

Diferencias significativas en el uso de las estrategias de modificación interna

\begin{tabular}{|c|c|c|c|}
\hline Estrategias & Función pragmática & Chino & Español \\
\hline $\begin{array}{l}\text { Marcadores de cortesía: } \\
\text { por favor (请/qLing), hacer } \\
\text { el favor (劳驾/láojià), etc. }\end{array}$ & $\begin{array}{l}\text { Se trata de expresiones } \\
\text { corteses y respetuosas que } \\
\text { sirven para conseguir la } \\
\text { cooperación del oyente. }\end{array}$ & & $\begin{array}{l}\text { S.4 ( } p=0.000) \\
\text { S.5 }(p=0.020) \\
\text { S.7 }(p=0.025) \\
\text { S.8 }(p=0.003) \\
\text { S.9 }(p=0.005) \\
\text { S.11 }(p=0.044)\end{array}$ \\
\hline $\begin{array}{l}\text { Minimizadores: un poco } \\
\text { (一点/yidiăn), alguno } \\
\text { (一些/yixiē), etc. }\end{array}$ & $\begin{array}{l}\text { Se trata de modificadores } \\
\text { que reducen la carga de la } \\
\text { fuerza ilocutiva. }\end{array}$ & $\begin{array}{l}S .1(p=0.049) \\
\text { S.5 }(p=0.010)\end{array}$ & \\
\hline $\begin{array}{l}\text { Marcadores pragmáticos } \\
\text { interpersonales: ¿ale? } \\
\text { (行吗 ? / xíng ma?, 可以 } \\
\text { 吗? / kěy } m a, \text { 好吗 ? / } \\
\text { hăo ma) }\end{array}$ & $\begin{array}{l}\text { El hablante apela al } \\
\text { consentimiento del oyente } \\
\text { de esta manera, y se sitúan } \\
\text { al final de la frase. }\end{array}$ & $\begin{array}{l}S .5(p=0.049) \\
S .7(p=0.041)\end{array}$ & \\
\hline $\begin{array}{l}\text { Reiteración de la petición: } \\
\text { Vámonos, vámonos en } \\
\text { seguida (Vivir: 172). }\end{array}$ & $\begin{array}{l}\text { La petición se reitera para } \\
\text { enfatizar la importancia } \\
\text { de la realización del acto } \\
\text { pedido. }\end{array}$ & & $\begin{array}{l}\text { S.3 }(p=0.010) \\
\text { S.5 }(p=0.042) \\
\text { S.7 }(p=0.001) \\
\text { S.9 }(p=0.042) \\
\text { S.11 }(p=0.009) \\
\text { S.12 }(p=0.027)\end{array}$ \\
\hline $\begin{array}{l}\text { Estructuras con } \\
\text { condicionales: si...(如 } \\
\text { 果.......的话/rúguǒ...... de } \\
\text { huà). }\end{array}$ & $\begin{array}{l}\text { La petición se basa en } \\
\text { condiciones hipotéticas } \\
\text { para atenuar la imposición } \\
\text { por parte del hablante. }\end{array}$ & & $\begin{array}{l}S .4(p=0.005) \\
S .6(p=0.048) \\
S .9(p=0.049) \\
S .10(p=0.006) \\
S .11(p=0.009) \\
S .12(p=0.003)\end{array}$ \\
\hline
\end{tabular}

Fuente: elaboración propia.

Otra observación fundamental que debemos realizar es que cada lengua muestra una preferencia de uso diferente. En español, la frecuencia es mayor 
en el uso de marcadores de cortesía en la mayoría de las situaciones. En chino, sin embargo, los interlocutores recurren más a estrategias propias, como es el caso de las siguientes:

a. Reductores de intensidad: son partículas que se sitúan al final de la frase para minimizar el impacto de la petición.

您让我上楼去吧。

Nín ràng wǒ shàng lóu qù ba $\underline{\text {. }}$

"Déjeme subir" (Yu, 2013, p. 26).

b. Reduplicación de verbos: repetición de los verbos. Sirve para reducir la fuerza ilocutiva.

先生的大作可-以拿-来看看吗?

Xiānsheng de dà zuò kè-y̌̌ ná-lái kàn kan ma?

“¿Puede mostrarnos su obra, señor?” (Qian, 2013, p. 205).

Este contraste se debe a la diferencia existente en la morfosintaxis, ya que tales estrategias sólo existen en chino, y es un factor importante que puede provocar el choque comunicativo en la comunicación intercultural, especialmente en el caso de los hispanohablantes cuando se comunican en chino con los sinohablantes, ya que no les es familiar el uso de los reductores de intensidad y de reduplicación de verbos. Por su parte, y debido a que en español no existen tales usos, los chinos recurrirán a otras estrategias que existen en ambas lenguas, por lo que en este sentido el problema será menos para ellos. En la enseñanza de las lenguas es necesario profundizar en este aspecto.

\subsection{Movimientos de apoyo}

Los movimientos de apoyo son elementos periféricos que pueden servir para que el oyente realice el acto pedido. En primer lugar, de acuerdo con la figura 3 , su uso es frecuente en todas las situaciones, excepto en la S.9 (D-, H = O, $\mathrm{G}+$ ), en que no ha llegado al 50.0\%. En español, sin embargo, sólo alcanza esta cifra en las S.4 (D+, H<O, G++), S.8 (D=, H<O, G++), S10 (D-, H = O, 
$\mathrm{G++}$ ) y S.12 (D-, H<O, G++). Como bien indica la siguiente figura, se observa una tendencia a un mayor uso en chino que en español, salvo en las S.4 (D+, $\mathrm{H}<\mathrm{O}, \mathrm{G++}$ ) y $\mathrm{S} .10(\mathrm{D}-, \mathrm{H}=\mathrm{O}, \mathrm{G}++$ ), y este contraste resulta significativo en tres situaciones: $1(\mathrm{D}+, \mathrm{H}=\mathrm{O}, \mathrm{G}+)\left(\mathrm{p}=0.004^{* *}\right), 3(\mathrm{D}+, \mathrm{H}<\mathrm{O}, \mathrm{G}+)(\mathrm{p}=$ $0.018)$ y $8(\mathrm{D}=, \mathrm{H}<\mathrm{O}, \mathrm{G}++)\left(\mathrm{p}=0.004^{* *}\right)$. De esto deducimos que en chino los interlocutores muestran una mayor conciencia de la intensidad a la hora de realizar la petición.

Figura 3

Frecuencia de uso de las modificaciones externas

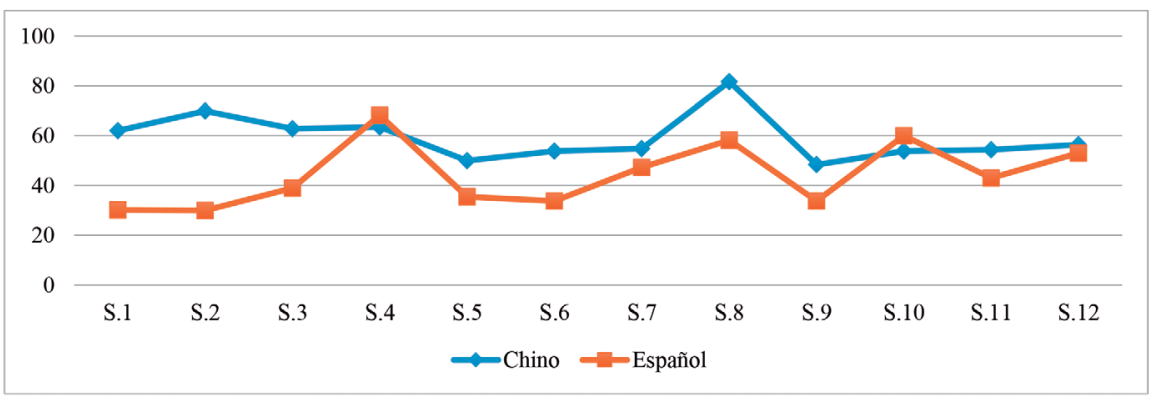

Fuente: elaboración propia.

En segundo lugar, en el análisis de la sensibilidad nuestra investigación ha arrojado los siguientes resultados:

1. El uso de las modificaciones externas no presenta sensibilidad a la distancia social. Es decir, sea cual sea la distancia social, no existen diferencias significativas en ambas lenguas.

2. Cuando el oyente tiene poder sobre el hablante, en español se emplean más modificaciones externas ( $\mathrm{P}=.000)$ : $34.1 \%$ frente a $50.3 \%$.

3. Tanto en chino como en español los interlocutores utilizan menos modificaciones externas cuando el grado de imposición es normal que cuando éste es alto:

Chino $(\mathrm{P}=.044): 55.1 \%$ frente a $62.4 \%$.

Español $(\mathrm{P}=.000): 39.0 \%$ frente a $54.2 \%$. 
En tercer lugar, cuando analizamos el uso de las estrategias concretas, podemos ver que en las dos lenguas se detecta la misma preferencia de uso de justificadores en las 12 situaciones. Las diferencias, por otro lado, resultan significativas en varios casos. Los preparadores y la interrogación para pedir la opinión del oyente son más habituales en español, así como el compromiso de recompensa en chino en las situaciones señaladas en la tabla 7 . En cuanto a los justificadores, se emplean más en la $\mathrm{S} .9(\mathrm{D}-, \mathrm{H}=\mathrm{O}, \mathrm{G}+$ ) en chino y en la S.3 $(\mathrm{D}+, \mathrm{H}<\mathrm{O}, \mathrm{G}+)$ en español.

\section{Tabla 7}

Diferencias significativas en el uso de estrategias de modificación externa

\begin{tabular}{|c|c|c|c|c|}
\hline Estrategias & Características & Ejemplos del corpus & Chino & Español \\
\hline Preparadores & $\begin{array}{l}\text { El hablante anticipa } \\
\text { la petición o } \\
\text { pregunta acerca de } \\
\text { la disponibilidad } \\
\text { potencial, el permiso, } \\
\text { la capacidad, etc., para } \\
\text { que el oyente lleve a } \\
\text { cabo el acto pedido. }\end{array}$ & $\begin{array}{l}\text { ¿Hay alguien que } \\
\text { sepa medicina? } \\
\text { ¡Venga algún } \\
\text { médico! } \\
\text { (Yan, 2012b, p. } \\
\text { 431). }\end{array}$ & & $\begin{array}{l}S .9(p=0.006) \\
S .12 \\
(p=0.011)\end{array}$ \\
\hline Justificadores & $\begin{array}{l}\text { El hablante expone } \\
\text { sus motivos, } \\
\text { explicaciones y } \\
\text { justificaciones para el } \\
\text { acto pedido. }\end{array}$ & $\begin{array}{l}\text { Date prisa, tengo } \\
\text { que ir a casa del } \\
\text { secretario Wang, en } \\
\underline{\text { la Aldea Norte, me }} \\
\underline{\text { ha llamado varias }} \\
\text { veces para apretar. } \\
\text { (Yan, 2012a, p. 18). }\end{array}$ & $\begin{array}{l}S .9 \\
(p=0.007)\end{array}$ & $\mathrm{S} .3(\mathrm{p}=0.017)$ \\
\hline $\begin{array}{l}\text { Compromiso } \\
\text { de recompensa }\end{array}$ & $\begin{array}{l}\text { El hablante promete } \\
\text { recompensas a cambio } \\
\text { de la realización del } \\
\text { acto pedido por parte } \\
\text { del oyente. }\end{array}$ & $\begin{array}{l}\begin{array}{l}\text { Suelta a mi } \\
\text { niño, voy a ser } \\
\text { responsable de todo. }\end{array} \\
\text { (Yan, 2012b, p. } \\
\text { 356). }\end{array}$ & $\begin{array}{l}S .4 \\
(p=0.007), \\
S .5 \\
(p=0.026) .\end{array}$ & \\
\hline $\begin{array}{l}\text { Interrogación } \\
\text { para pedir } \\
\text { opinión }\end{array}$ & $\begin{array}{l}\text { Para que la petición } \\
\text { sea más aceptable, el } \\
\text { hablante consulta la } \\
\text { opinión del oyente. }\end{array}$ & $\begin{array}{l}\text { ¿Quiere que nos } \\
\text { tuteemos? Yo creo } \\
\text { que ya debemos } \\
\text { tutearnos, ¿no te } \\
\text { parece? } \\
\text { (Cela, 2001, p. 186). }\end{array}$ & & $\mathrm{S} .8(\mathrm{p}=0.040)$ \\
\hline
\end{tabular}

Fuente: elaboración propia. 
En la tabla anterior observamos que la estrategia del compromiso de recompensa presenta un mayor uso en las $\mathrm{S} .2(\mathrm{D}+, \mathrm{H}=\mathrm{O}, \mathrm{G}++), \mathrm{S} .3(\mathrm{D}+, \mathrm{H}<$ $\mathrm{O}, \mathrm{G}+)$ y $\mathrm{S} .4(\mathrm{D}+, \mathrm{H}<\mathrm{O}, \mathrm{G}++)$, en las que el riesgo potencial del FTA puede ser relativamente más alto. Según la máxima de tacto que establece Leech (1983, p. 119) - minimice el coste para el otro, maximice el beneficio para el otro-, dicha estrategia resulta relativamente más cortés, en comparación con otras, ya que ofrece directamente un beneficio a cambio del acto pedido. En este sentido, podemos decir que los interlocutores han elegido el uso del compromiso de recompensa porque tienen más necesidad de mitigar la fuerza ilocutiva del acto principal en esas situaciones.

En los análisis de las modificaciones externas hemos visto que la estrategia de autodenigración - que se refiere a que el hablante se echa la culpa o se denigra a sí mismo para suscitar la compasión del oyente o para mostrar modestia - se ha utilizado en la mitad de las situaciones en chino, como sucede en el siguiente ejemplo.

\section{我_二=妇-道_人-家-本-不该多嘴, 你 能 不 能}

Wǒ yí-gè fùdào rén jiā běn bù gāi duō zuľ, nǐ néng bù néng

放-了他-们？怎-么-着他-们也是你-的姐-夫妹-夫小-姨-子。

fàng-le tā-men? zěn-me-zhe tā-men yě shì nǐ-de jiě-fū mèi-fū Xiăo-yí-zi.

"Yo sólo soy una mujer y no debo comentar más, ¿puedes dejarlos en libertad? De todas maneras, ellos son tus cuñados y cuñada” (Yan, 2012b, p.245).

Como destaca Gu (1990), dicha estrategia es una de las máximas de cortesía en chino; más aún, es la más específica y característica en dicha cultura, y, en consecuencia, no hemos encontrado ningún caso de su uso en español.

En la vida cotidiana solemos ver que los sinohablantes suelen rechazar el elogio de los occidentales. Por ejemplo, cuando un hispanohablante le dice a un estudiante chino: Habla muy bien usted el español, en vez de aceptarlo diciendo Gracias, ellos lo rechazarán con expresiones del tipo de: ¡Qué va! Hablo muy mal o No, aún me falta mucho, hecho que puede resultar descortés para los hispanohablantes, ya que amenaza la imagen positiva del hablante -deseo de ser apreciado por los demás y de que otros compartan los mismos deseos (Brown \& Levinson, 1987)—. Sin embargo, en chino se considerará 
cortés, ya que también es un modo de expresar la modestia. La estrategia de autodenigración, al tratarse de una característica exclusiva china, puede provocar fácilmente choques culturales en la comunicación chino-española.

Sintetizando lo anterior, los resultados obtenidos demuestran que las diferencias se observan tanto en el uso general de modificaciones externas como en el empleo de estrategias concretas, y esto se debe no sólo a las diferencias intralingüísticas, sino también a la percepción del concepto de cortesía, origen del choque comunicativo intercultural.

\subsection{Marcadores de apertura}

Los marcadores de apertura sirven principalmente para llamar la atención del oyente antes de emitir la petición, ya que si el oyente no recibe la información, el acto de habla falla.

En las 12 situaciones, como ilustra el siguiente gráfico, en chino el uso de marcadores de apertura ha sobrepasado el $50 \%$ en más de la mitad de las situaciones; sin embargo, en español es escaso. Este contraste resulta llamativo, salvo en la S. $1(\mathrm{D}+, \mathrm{H}=\mathrm{O}, \mathrm{G}+)$ y la $\mathrm{S} .10(\mathrm{D}-, \mathrm{H}=\mathrm{O}, \mathrm{G}++)$.

\section{Figura 4}

Frecuencia de uso de los marcadores de apertura

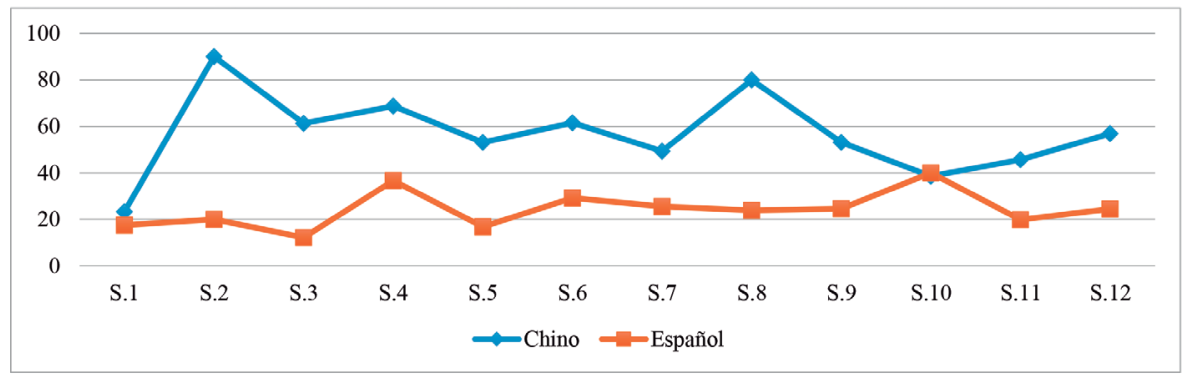

Fuente: elaboración propia.

Al analizar la sensibilidad que muestran los marcadores de apertura, vemos que:

1. Sólo el chino muestra sensibilidad a la distancia social $(\mathrm{P}=.049)$ : cuando la distancia social es mayor, los interlocutores utilizan más marcadores de apertura que cuando ésta es menor. En cambio, en español su uso no ha sido influido por las diferentes categorías de la distancia social. 
2. El uso de los marcadores de apertura es indiferente al poder relativo en las dos lenguas.

3. Tanto en chino como en español, cuando el grado de imposición es normal, los interlocutores utilizan menos marcadores de apertura que cuando éste es alto:

Chino $(\mathrm{p}=.005), 53.7 \%$ frente a $63.7 \%$.

Español $(p=.028), 20.1 \%$ frente a $27.9 \%$.

En cuanto a la diferencia en el uso de las estrategias, el análisis estadístico muestra que las fórmulas que denotan respeto, las de tratamientos nominales y las relativas al parentesco se utilizan con mayor frecuencia en chino; el uso de las fórmulas de llamada de atención es más habitual en español en las situaciones indicadas en la tabla 6.

\section{Tabla 8}

Diferencias significativas en el uso de las estrategias de marcadores de apertura

\begin{tabular}{|c|c|c|c|}
\hline & Características & Chino & Español \\
\hline $\begin{array}{l}\text { Fórmulas de } \\
\text { tratamiento } \\
\text { de respeto (de } \\
\text { formalidad) }\end{array}$ & $\begin{array}{l}\text { Pueden ser tratamientos honoríficos } \\
\text { como el apellido + cargo, por } \\
\text { ejemplo: gerente Wang (王总/Wáng } \\
\text { zǒng). }\end{array}$ & $\begin{array}{l}S .3(p=0.022) \\
S .4(p=0.002) \\
S .5(p=0.017)\end{array}$ & \\
\hline $\begin{array}{l}\text { Fórmulas de } \\
\text { tratamiento } \\
\text { nominales }\end{array}$ & $\begin{array}{l}\text { Se trata principalmente de los } \\
\text { nombres del oyente. }\end{array}$ & $\mathrm{S} .12(\mathrm{p}=0.044)$ & \\
\hline $\begin{array}{l}\text { Fórmulas de } \\
\text { tratamiento } \\
\text { relativas al } \\
\text { parentesco }\end{array}$ & $\begin{array}{l}\text { Se trata de apelativos que indican la } \\
\text { relación familiar: tío (叔父/shūfü), } \\
\text { hermana mayor (姐姐/jiějie), etc. }\end{array}$ & $\begin{array}{l}S .5(p=0.031) \\
S .8(p=0.037) \\
S .11(p=0.031) \\
S .12(p=0.013)\end{array}$ & \\
\hline $\begin{array}{l}\text { Fórmulas de } \\
\text { llamada de } \\
\text { atención }\end{array}$ & $\begin{array}{l}\text { Son fórmulas que sirven para llamar } \\
\text { directamente la atención del oyente, } \\
\text { como oye (喂/wèi). }\end{array}$ & & $\begin{array}{l}S .3(p=0.000) \\
\text { S.4 }(p=0.000) \\
\text { S.7 }(p=0.001) \\
\text { S.9 }(p=0.010)\end{array}$ \\
\hline
\end{tabular}

Fuente: elaboración propia. 
Observamos en el corpus que las fórmulas de tratamiento forman una gran parte de las estrategias de los marcadores de apertura. En realidad, la función de éstas no sólo se limita a llamar la atención de los oyentes, sino que también implica una serie de informaciones sociolingüísticas.

En chino existen dos grandes clases de fórmulas de tratamiento $(\mathrm{Qu} \&$ Chen 1999, p.119):

1. Fórmulas de tratamiento basadas en la máxima de intimidad.

En la cultura china, el valor del reconocimiento de sí mismo se basa en la relación interpersonal interdependent self, por lo que no hay un límite claro entre personas, dependen de sí mismos y se evalúan mutuamente (Qu y Chen, 1999, p. 119), muy diferente al mundo occidental, en el que se depende de sí mismo independent self. Esta dependencia interpersonal determina que la intimidad sea un factor importante en la percepción del concepto de la cortesía: cuanta más intimidad se demuestra en el acto verbal, más cortés se considerará. En consecuencia, el uso de apelativos relativos al parentesco es común en chino, ya que resulta pragmáticamente cortés y puede aplicarse en cualquier tipo de relación: conocidos, desconocidos y familiares. Efectivamente, este tipo de fórmulas de tratamiento han aparecido en la mayoría de las situaciones, como se ve en el siguiente ejemplo de la S.2 ( $+, \mathrm{H}=\mathrm{O}, \mathrm{G}++)$, donde los interlocutores son desconocidos o han tenido poca relación social:

好- 大-哥, 你要怎-么-样都行, 只求你能 把

Hăo dà-gē, nǐ yào zěn-me-yàng dōu xíng, zhǐ qiú nǐ néng bă

俺赶-快送出-去, 俺家里还有个吃奶的娃。

ăn găn-kuài sòng chū-qù, ăn jiā lǐ hái yǒu gè chī năi de wá

"Amable hermano, puedes hacer lo que quieras conmigo, sólo te suplico que me saques de aquí en seguida, tengo un bebé lactante en casa" (Yan, 2012b, p.597).

En español, sin embargo, las fórmulas de tratamiento relativas al parentesco en español son casi exclusivas para los familiares o parientes. 
2. Fórmulas de tratamiento basadas en la máxima de relación social. Debido a que el valor del reconocimiento de sí mismo se basa en la relación interpersonal, los sinohablantes estiman en mayor grado la evaluación de los demás, por lo que en consecuencia cuanto más elevamos al receptor mediante expresiones verbales, más cortés resulta el acto. En correspondencia con esto, en el corpus hemos observado un fenómeno muy minoritario en el español: los chinos suelen referirse al interlocutor mediante su cargo, hecho que puede reconocer la reputación o el prestigio que se obtiene en la vida mediante el éxito y la ostentación, reconociendo así el estatus del oyente. De esta manera, el oyente también puede conseguir el valor del reconocimiento de sí mismo. Como hemos dicho anteriormente, aunque en español también se observa un aspecto semejante, no es tan habitual como en chino.

\section{校-长, 别 开-除我, 别 开-除我。}

Xiào-zhăng, bié kāi-chú wǒ, bié kāi-chú wǒ.

“Director, no me expulse, no me expulse" (Yan, 2012a, p.45).

Como hemos visto, el valor de la cortesía verbal en las dos culturas da lugar directamente a las diferencias en el uso de las fórmulas de tratamiento. Debido a que el uso apropiado de estas fórmulas se considera directamente una máxima de cortesía, tienen un peso pragmático más importante en la cultura china que en la española. Puede estar vinculado a este aspecto el hecho de que en chino se observe una gran variedad de fórmulas de tratamiento, mientras que en español se vea relativamente más homogénea.

\section{Conclusión}

En primer lugar, según los resultados, los interlocutores de ambas lenguas muestran cierta conciencia del riesgo potencial del FTA y la distancia social en los actos principales, pero hay que resaltar que en español se muestra más sensibilidad a esta variable. Esto nos indica que los interlocutores de ambas lenguas tienen en cuenta la distancia social en los procesos cognitivos de la realización de la petición, por lo que pueden aplicar estrategias diferentes según las relaciones interpersonales: desconocidos, conocidos e íntimos. Sin 
embargo, el poder relativo y el grado de imposición no tienen un impacto tan importante. Si la distancia social influye cognitivamente sobre la petición, estas dos variables pueden influir de manera mecánica o rutinaria. Es decir, cuando no hace falta considerar la distancia social, los interlocutores pueden elegir estrategias que suelen utilizar o que son comunes en situaciones determinadas. En este sentido, el chino y el español muestran la misma propiedad pragmática.

En segundo lugar, cuando la distancia social es mayor, los interlocutores de ambas lenguas utilizan más modificaciones internas que cuando ésta es neutra o menor, por lo que su uso es bastante sensible a la distancia social tanto en chino como en español. Sin embargo, el uso de las modificaciones internas sólo es frecuente en chino, en la mayoría de las situaciones y cualquier categoría de las tres variables sociales en chino; en español, por su parte, se muestra un uso reducido. La conclusión a la que llegamos es que los sinohablantes suelen recurrir más a las modificaciones internas que los hispanohablantes. La preferencia de su uso en chino puede ser una propiedad lingüística específica, sea por la costumbre pragmática o por la gramática. En cuanto a las modificaciones externas, al igual que el caso anterior, su uso es más común en chino, y el grado de imposición es el factor más influyente en ambas lenguas.

Por último, en lo que respecta al uso de marcadores de apertura, podemos destacar que se trata de otro aspecto muy diferente, ya que en más de la mitad de las situaciones ha superado el $50.0 \%$ en chino, hecho que no se observa en español. Por otro lado, las dos lenguas muestran sensibilidad al grado de imposición, ya que cuando el grado de imposición es normal, los interlocutores utilizan menos marcadores de apertura que cuando éste es alto. Sin embargo, sólo el chino muestra sensibilidad a la distancia social: cuando la distancia social es mayor, los interlocutores utilizan más marcadores de apertura que cuando ésta es menor.

Los resultados anteriores podrán contribuir, por un lado, a mejorar la comunicación intercultural entre el español y el chino, evitando errores de transferencia pragmática de una a la otra, teniendo en cuenta los aspectos contrastivos. Por otro, también ofrecerá argumentos sólidos que demuestran que se debe prestar especial atención en las traducciones literarias chinoespañolas, ya que debido a la falta de competencia pragmática, los contenidos interpretados en otra lengua pueden diferir de los originales. 


\section{Referencias}

Amzilotti, G. I. (1983). Searching for linguistic universals through contrastive analysis. Cross-language Studies, 10, 1-17. https://doi.org/10.1017/ S0261444800004936

Binti, R. I. (2013). Análisis contrastivo de la cortesía verbal en Español y Malayo en la realización de tres actos de habla; petición, mandato y rechazo (Tesis sin publicar). Universidad de Valladolid.

Briz, G. E. A. (2010). La cortesía al hablar español. Beijing: III Jornadas de formación de profesores de E/LE. http://www.sinoele.org/images/Revista/3/ iiijornadasP_Briz.pdf

Brown, P., \& Levinson, S. (1994). Politeness. Some universals in language usage. Cambridge University Press.

Cela, C. J. (2001). La colmena. Cátedra.

Coulmas, F. (1981). Conversational routine: Explorations in standardized communication situations and prepatterned speech. Mouton.

Gu, Y. (1990). Politeness in modern Chinese. Journal of Pragmatics, 14(2), 237-257. https://doi.org/10.1515/ip-2018-0024

Kitagawa, C. (1980). Saying "Yes" in Japanese. Journal of Pragmatics, 4(2), 105-120. https://doi.org/10.1016/0378-2166(80)90048-X

Leech, G. N. (1983). Principles of pragmatics. Longman.

Qian, Z. S. 钱钟书. (2013). 围城 [La fortaleza asediada]. 人民文学出版社 [People's Literature Publishing House].

Qu, W., \& Chen, S. (1999). Explicación de las fórmulas de tratamiento corteses. Journal of East China Normal University, 6, 118-124.

Rue, Y. J., \& Zhang, G. Q. (2008). Request Strategies: A Comparative Study in Mandarin Chinese and Korean. John Benjamins Publishing Company.

Sánchez, R. F. (2006). El Jarama. Pozuelo de Alarcón.

Tzu, L. Y. (2015). Estudio pragmalingüístico español y chino de actos de habla expresivosdisculpas y agradecimiento (Tesis sin publicar). Universidad de Valladolid.

Yan, M. 莫言. (2012a). 天堂赫荎之歌 [Las baladas del ajo]. 作家出版社 [Writers Publishing House].

Yan, M. 莫言. (2012b). 丰乳肥慰 [Grandes pechos, amplias caderas]. 作家出 版社 [Writers Publishing House].

Yu, C. 曹禺. (2013). 雷雨 [La Tempestad]. 人民文学出版社 [People's Literature Publishing House]. 